\title{
Performance evaluation of cymbal hydrophones for underwater applications
}

\author{
C. Kannan, R. Dhilsha*, P. M. Rajeshwari, Shibu Jacob, M. A. Atmanand \\ National Institute of Ocean Technology, Ministry of Earth Sciences, Pallikaranai, Chennai-600 100, Tamil Nadu, India
}

Email address:

krd@niot.res.in(R. Dhilsha)

\section{To cite this article:}

C. Kannan, R. Dhilsha, P. M. Rajeshwari, Shibu Jacob, M. A. Atmanand. Performance Evaluation of Cymbal Hydrophones for Underwater Applications. International Journal of Mechanical Engineering and Applications. Vol. 1, No. 2, 2013, pp. 43-48. doi: $10.11648 /$ j.ijmea.20130102.13

\begin{abstract}
This paper reports the performance differences in the receiving sensitivity and band width of Class $\mathrm{V}$ flex tensional transducers (Cymbals) both in-air and in-water by varying the design parameters. Cymbal elements with device diameter $8 \mathrm{~mm}$ and $13 \mathrm{~mm}$ with optimum designs achieved using finite element software ATILA is presented. The in-air and underwater characteristics of such transducers are described in this paper. The performance evaluation of the transducers both in air and water are also reported in this paper. It has been established that the resonance frequency shifts towards higher side while increasing the cavity depth retaining the cap diameter fixed. However, there exists a tradeoff between the choice of sensitivity and optimum cavity depth. A maximum Receiving Sensitivity (RS) of -203 dB re 1V/ $\mu \mathrm{Pa} @ 1 \mathrm{~m}$ is obtained analytically for a single element with $13 \mathrm{~mm}$ diameter and a cavity depth of 600 micron with a band of 2-20 kHz. Also a wide band of $1-30 \mathrm{kHz}$ is achieved with $8 \mathrm{~mm}$ cymbal element having the same cavity depth with a Receiving Sensitivity $-202.84 \mathrm{~dB}$ re $1 \mathrm{~V} / \mu \mathrm{Pa} @ 1 \mathrm{~m}$. Effect of encapsulation on the performance of cymbal transducers is studied and analytical results are in agreement with experimental results. There exist critical fabrication factors that affect the performance of these elements while fabricated in bulk quantities for an array. Reliable production procedure has been developed for manufacturing of cymbal elements and its arrays. A $1 \times 3$ array of $13 \mathrm{~mm}$ diameter cymbal transducer has been fabricated and tested for in-air and in-water performances. The RS showed a maximum value of $-178 \mathrm{~dB}$ re $1 \mathrm{~V} / \mu \mathrm{Pa} @ 1 \mathrm{~m}$ in a frequency range of 2-20 kHz with an in-house developed preamplifier having a gain of $20 \mathrm{~dB}$ for a single element using brass as cap material. An array gain of nearly $6 \mathrm{~dB}$ is obtained when connected in series. The procedure adopted for the fabrication of Cymbal elements is critical for obtaining a flat hydrophone array response over a wide frequency range.
\end{abstract}

Keywords: Cymbal Transducers, Hydrophones

\section{Introduction}

Flex-tensional transducers have wide range in size from several millimeters to fraction of a meter in length and can weigh up to hundreds of kilograms. A miniaturized version of the class $\mathrm{V}$ flex-tensional transducer called the Moonie was first developed at the Material Research Laboratory at the Pennsylvania State University in the late 1980's and cymbal was first evolved from the Moonie in 1998 and patented by Newnham and Dogan [1]. Extensive studies were done by Newnham et al on the effect of ceramic diameter, thickness and cavity depth of end cap, cavity diameter and material properties of end caps and ceramics on the first resonance frequency of the cymbal transducer. Newnham et al has used a standard cymbal transducer with a diameter of $12.7 \mathrm{~mm}$ and a cavity depth of $0.25 \mathrm{~mm}$ over a wide frequency range throughout the paper with Titanium end cap material. Newnham et al has reported cymbal transducers with a wide range of frequency from $5 \mathrm{kHz}-$ $150 \mathrm{kHz}$ in water by varying the dimensions and types of materials [2]. After that several studies were made on the cymbal type flex-tensional transducer by several groups including NIOT [8-10]. In this paper, an improved $13 \mathrm{~mm}$ diameter cymbal transducer with 600 micron cavity depth and $0.15 \mathrm{~mm}$ metal cap thickness having low frequency of operation, $2 \mathrm{kHz}-20 \mathrm{kHz}$ is presented. Also analytical studies were made on $8 \mathrm{~mm}$ cymbal transducer to increase the frequency band of operation. Moreover brass is used here as cap material. Due to the reversibility of piezoelectric effect, cymbal transducer can be used as both underwater sound projector and receivers. The miniaturized nature combined with the flexibility in the choice of frequency, 
band width, medium power ( $20 \mathrm{~dB})$ and flexibility to use in the design and fabrication of planar and conformal array made them attractive for AUV applications compared to conventional transducers. Even though the transducer element looks simple, the design and development of the same for underwater applications involve a lot of challenges for reliable repeatable results. There are several factors that affect the performance of such an underwater transducer when compared to air applications. The major factors which affect the performance are piezo electric properties of the ceramics, diameter and thickness of the ceramic, mechanical properties of end cap, mechanical properties gluing material, cavity depth, thickness of metal cap and gluing techniques. For hydrophones, the most important parameters required for characterizations are resonance frequency $\left(f_{r}\right)$, mechanical quality factor $\left(\mathrm{Q}_{\mathrm{m}}\right)$, electro mechano-acoustic efficiency $\eta_{\text {ma }}$, electromechanical coupling coefficient $\left(k_{\text {eff }}\right)$, acoustic directivity and Free Field Voltage Sensitivity (FFVS) [2] Cymbal hydrophone can be made to perform over a wide bandwidth without much reduction in sensitivity by optimizing the Cavity Depth, sheet thickness, diameter of cavity and the selection of piezo-ceramic. Considering above parameters, cymbal elements with good Receiving Sensitivity (RS) and wide band has been reported by D. Li et al [3]. Results of a hydrophone, manufactured based on ATILA model results, fabricated using precision tools developed and indigenous material components are presented in this paper. Several iterations were made by varying the parameters using the finite element software ATILA for optimizing the transducer materials and dimensions. A preamplifier has also been developed to obtain an enhanced linear response with $\sim 20 \mathrm{~dB}$ gain.

\section{Finite Element Model and Comparison with Numerical Calculations}

Finite element analysis software ATILA was used to model the transducer element and to predict the air /underwater performances. Several iterations were done to optimize the dimensions and materials. Principle of axi-symmetry is used while modeling the element to reduce the number of nodes and elements and thereby the computation time. Structured meshing pattern with Quadrilateral elements were used for meshing and an optimum mesh of a $13 \mathrm{~mm}$ cymbal transducer element is shown in Fig.1.

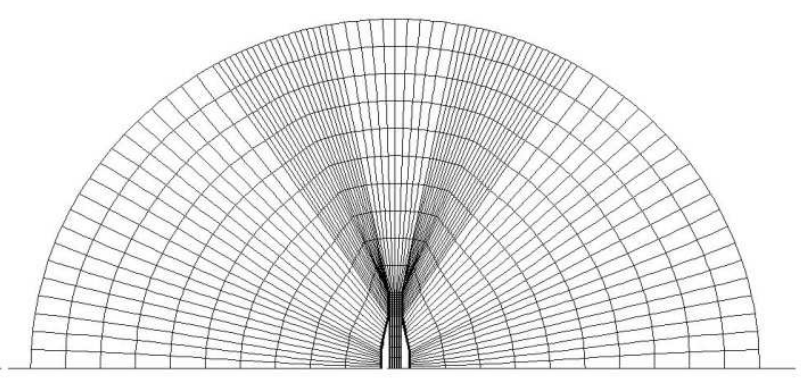

Figure 1. Structured mesh of a single Cymbal element with water

5141 nodes were used in the model with a total of 1650 Quadrilateral elements. Indigenous PZT-5A ceramics were used as active material and commercially available ordinary brass sheet was chosen for the end cap material for $13 \mathrm{~mm}$ cymbal elements. A cross sectional view of optimized cymbal hydrophone is shown in Fig.2. Fig.3 shows the simulated results showing the receiving sensitivity (RS) verses frequency of $13 \mathrm{~mm}$ cymbal by varying cavity depths.

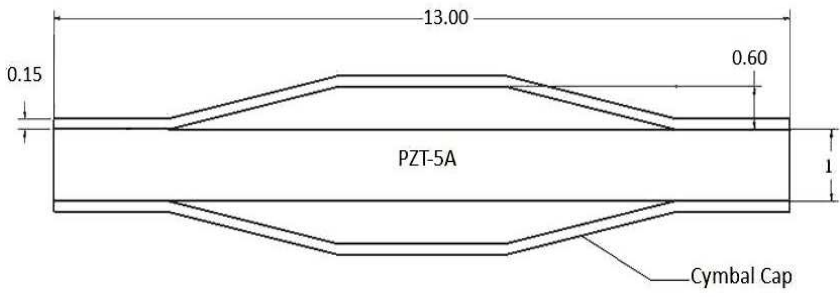

Figure 2. Cross section of cymbal hydrophone (All dimensions are in mm)

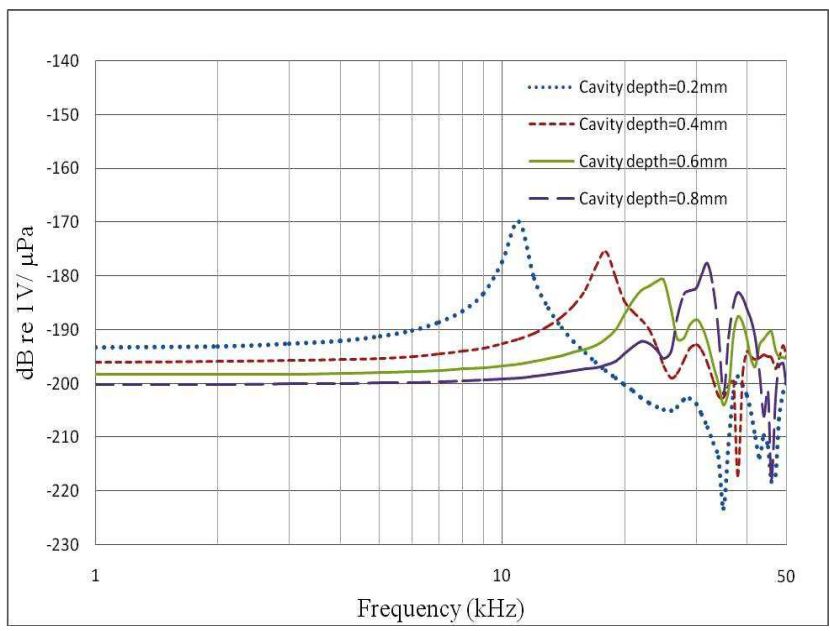

Figure 3. Simulated Receiving sensitivity of $13 \mathrm{~mm}$ cymbal hydrophone

It is observed that the RS values vary from -193 to -202 $\mathrm{dB}$ re $1 \mathrm{~V} / \mu \mathrm{Pa}$ over a frequency range of $1-20 \mathrm{kHz}$ while varying the cavity depth using pure brass as the cap material. It shows an increase in resonance frequency with an increase in cavity depth. Even though the elements with a cavity depth of 200 micron is giving more receiving sensitivity, it is feasible to opt the element with 600 micron for the hydrophone in order to get the required band of $2-20 \mathrm{kHz}$. So, a cymbal element with diameter $13 \mathrm{~mm}$ and a cavity 
depth of 600 micron having receiving sensitivity of $-198 \mathrm{~dB}$ re $1 \mathrm{~V} / \mu \mathrm{Pa}$ is found to be the optimum design for a hydrophone with the $13 \mathrm{~mm}$.

Fig.4 shows simulated Receiving sensitivity for $8 \mathrm{~mm}$ cymbal hydrophone elements obtained using ATILA software. RS curves are plotted for different cavity depths keeping all other parameters constant. The caps are $8.0 \mathrm{~mm}$ in diameter with a base cavity diameter of $4.0 \mathrm{~mm}$ and an apex diameter of $1.5 \mathrm{~mm}$. It is clear from the curves that the Receiving sensitivity is shifting towards the higher frequency as the cavity depth is increased resulting in higher band width. A wide band of $1-30 \mathrm{kHz}$ is observed with $8 \mathrm{~mm}$ cymbal element having the a cavity depth of 600 micron with a Receiving Sensitivity $-202.84 \mathrm{~dB}$ re $1 \mathrm{~V} / \mu \mathrm{Pa} @ 1 \mathrm{~m}$.

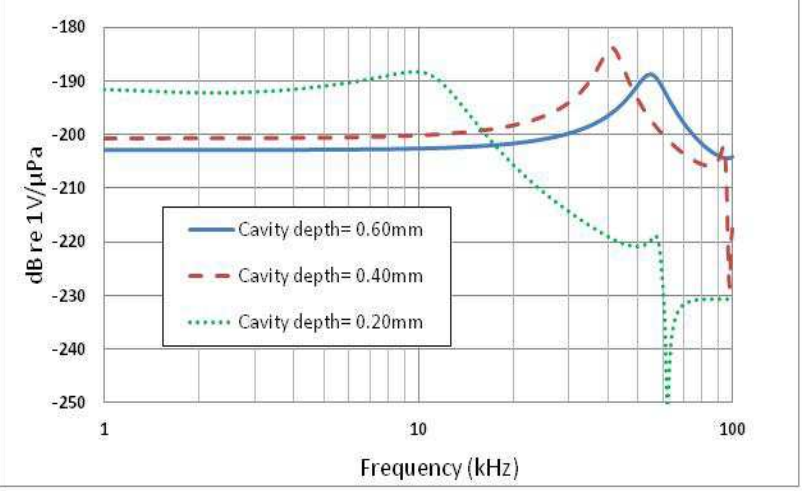

Figure 4. Simulated Receiving sensitivity for $8 \mathrm{~mm}$ cymbal hydrophone elements

Initial modeling has been done without considering the insulating epoxy. The use of insulating epoxy was found to affect the performances of cymbal transducers.

Modeling has been repeated after considering, a thin layer of $3 \mathrm{M}$ Scotchcast $2140 \mathrm{U}$ Resin with a thickness of $3 \mathrm{~mm}$ upon the flat portion of the metal cap. From the ATILA results, it is observed that by increasing the thickness of the cap layer, there is a significant decrease in RS values. Fig.5 shows the simulated impedance/phase angle variation of cymbal transducer in air both before and after encapsulation as a function of frequency. It is also observed that the resonance frequency has been shifted from $45 \mathrm{kHz}$ to $39 \mathrm{kHz}$ during encapsulation. In-air resonance frequency of cymbal transducer is at $45 \mathrm{kHz}$. In-water resonance is occurring at a frequency of $24 \mathrm{kHz}$ as per ATILA results and is expected due to water loading. It is also to be noted that the effect of gluing is not considered here while modeling because of the difficulty in the meshing. This may lead to a mismatch between the ATILA and experimental results.

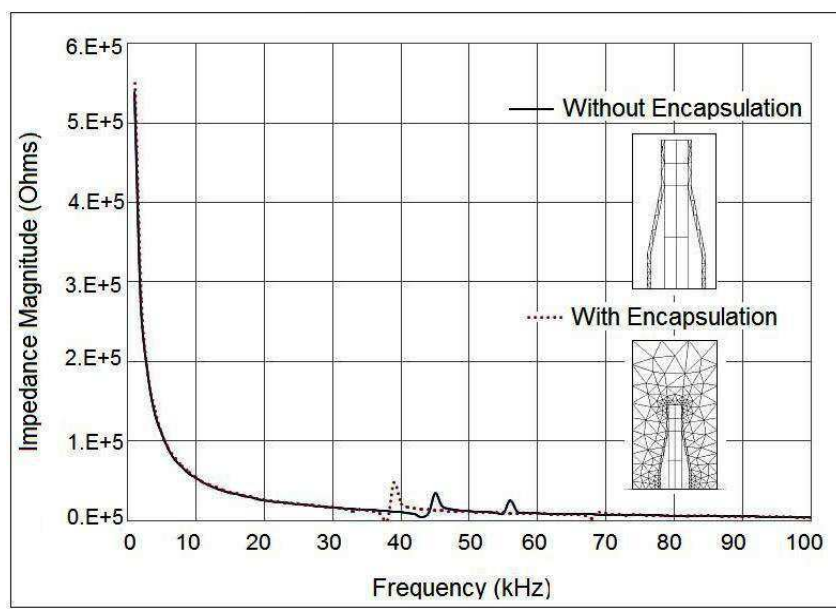

Figure 5.Simulated Impedance of cymbal transducer in air both before and after encapsulation

The fundamental resonance frequency in air is governed by end cap dimensions and elastic constants. This is obtained from the relation. [4].

$$
f_{r} \propto \sqrt{\frac{E}{\rho_{c}}\left[\frac{1}{d^{2}\left(1-\sigma^{2}\right)}+\frac{1}{R^{2}}\right]}
$$

But, in-water resonance

$$
\mathrm{f}_{\mathrm{rw}}=\frac{\mathrm{f}_{\mathrm{r}}}{\sqrt{1+\left(\frac{4 \mathrm{~d}}{3 \pi}\right)\left(\frac{\rho_{\mathrm{w}}}{\rho_{\mathrm{c}}}\right)\left(\frac{0.7885}{\mathrm{t}_{\mathrm{m}}}\right)}}
$$

where $t_{m}$ is the end cap thickness.

The coefficient 0.7885 is calculated by assuming boundary conditions based on curve deflection theory for a thin plate clamped around its rim [5]. In the particular case, the denominator in the right hand side of equation (2) will become 1.845 which corresponds to the geometry of the transducer. So the in-water resonance frequency, $\mathrm{f}_{\mathrm{rw}}$ will be $24 \mathrm{kHz}$. The predicted value using the theory has been obtained as well.

\section{Experimental Results and Discussions}

Cheng-Liang Sun et al [6] reported that, the ceramic can generate a constant force under the same electric field, but when the ceramic is sandwiched between two end caps, the end caps may restrict the strain produced by the ceramic. Therefore the experimental results are expected to be lower than the numerical results. The ATILA modeling results are in good agreement with the experimental results. Design and development of cymbal transducer requires precision manufacturing of the metal caps, precision tools and a production procedure with skill in the manufacturing and assembly is required in order to achieve desired performance. A specially designed combination die setup has been fabricated for carrying out the blanking and forming operation simultaneously at a single stroke. A hydraulic press with a maximum capacity of 50 Tones was used for delivering the required blanking and forming force such that 
the shearing and cracking will happen at the edges as well as metal will form to the designed 'cymbal' like shape. The ceramic discs were tested for its dynamic strain coefficient $\mathrm{d}_{33}$ values using Piezo Test PM-200, a digital piezo-meter. OptoNCDT 1605, a non contact laser displacement sensor is used for the measurement of cavity depth. Metal caps with exact dimensions are selected from the caps which are produced after the blanking and the forming procedure using the specially designed combination die setup. The flat periphery of the metal caps was then roughed using a double-cut flat needle file without affecting the dimensions and geometry of the formed metal cap. Both the surfaces of the ceramic and the periphery of the metal caps should be rough and clean for the proper bonding between the ceramic and the metal cap. The caps were then bonded to the piezoelectric ceramic disc. The ceramic disc and the end caps were bonded together around the circumference with glue and a constant pressure was applied over the bonding area using a specially designed jig. Art of applying glue in between metal caps and ceramics is very critical in obtaining constant transducer performances. If the bonding is not proper, non uniform bonding layer causes de-bonding during testing which lead to low $\mathrm{d}_{33}$ values [7]. Careful gluing with good skill was ensured for improved/enhanced performance. Most of the work reported in literature has been carried out using Titanium metal caps where thermal expansion coefficients of the metal and ceramics are likely to affect the performance.

With the optimized design, a $13 \mathrm{~mm}$ cymbal linear array $(1 \times 3)$ has been fabricated and the in-air impedance of the array was measured using an Agilent 4194A impedance analyzer. The first resonance of the fabricated device before encapsulation was obtained at $42.09 \mathrm{kHz}$ with an impedance of $2.07 \mathrm{k} \Omega$ along with a phase of $\left(-82.8^{0}\right)$ as shown in Fig.6.

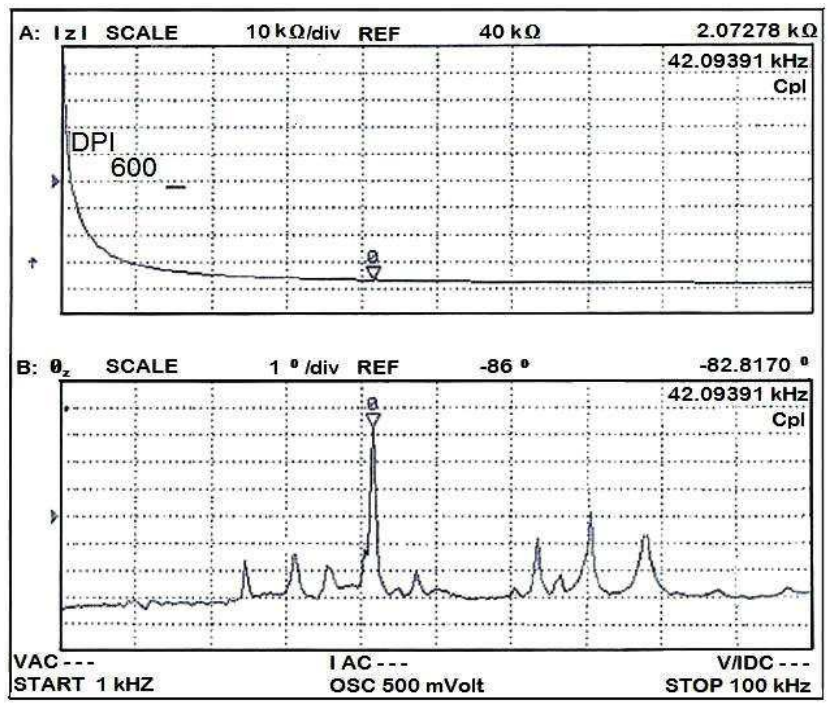

Figure 6. Measured impedance and phase of $13 \mathrm{~mm}$ diameter cymbal hydrophone with 600 micron cavity depth as a function of frequency
$2140 \mathrm{U}$ Scotchcast resin, the resonance frequency has been shifted to $36 \mathrm{kHz}$, and matching well with the ATILA model results. In-water parameter evaluations were carried out at $3 \mathrm{~m}$ water depth in the frequency range $2-25 \mathrm{kHz}$ at Acoustic Test Facility (ATF), NIOT. This facility has obtained an ISO/IEC 17025:2005 accreditation from National Accreditation Board for Testing and Calibration Laboratories (NABL) for testing and calibration parameters of underwater acoustic transducers. This facility consists of an Acoustic Tank, Acoustic Calibration System (ACS) and Acoustic Transducer Positioning System (ATPS). Acoustic transducers are characterized using comparison calibration and reciprocity calibration techniques. It is equipped with a full complement of both digital and analog test equipment, capable of performing Receiving sensitivity, Transmitting Voltage/Current Response, Transmit and Receive Beam Patterns, Impedance/Admittance/Phase versus frequency etc. The experiments were carried out, satisfying far field conditions. A maximum sensitivity of $-198 \mathrm{~dB}$ re 1V/ $\mu \mathrm{Pa} @$ $1 \mathrm{~m}$ was obtained experimentally, with its first resonance at $24 \mathrm{kHz}$. The sensitivity is approximately constant up to a frequency of $18 \mathrm{kHz}$. Comparison between model simulation and experimental results for a $13 \mathrm{~mm}$ element with a good dynamic strain coefficient $\mathrm{d}_{33}$ (15000 Pico coulomb) is shown in Fig.7

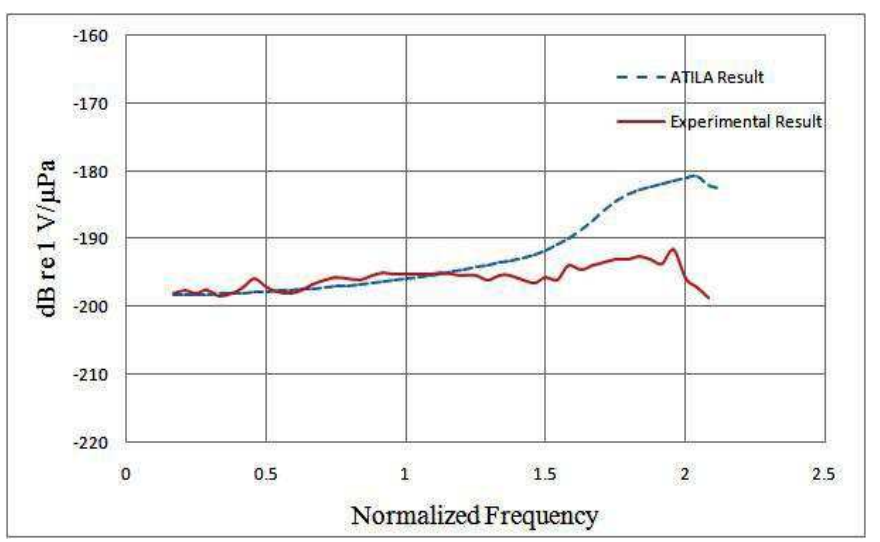

Figure 7. Comparison of Receiving Sensitivity with ATILA model and experimental results

A deviation at the tail end portion of the graph is mainly due to some of the assumptions made while modeling the cymbal element in ATILA. The glue area was not considered at the time of modeling and the edges of the metal caps were considered as sharp a corner which is not so in reality .The $\mathrm{d}_{33}$ values were found to vary from $3000-15000$ if the fabrication techniques are not uniform. Measured sensitivity of $13 \mathrm{~mm}$ diameter PZT-5A driven cymbal hydrophone array with a variation in $d_{33}(1 \times 3)$ with linear Preamplifier gain of $20 \mathrm{~dB}$ gain is shown in Fig. 8 . 


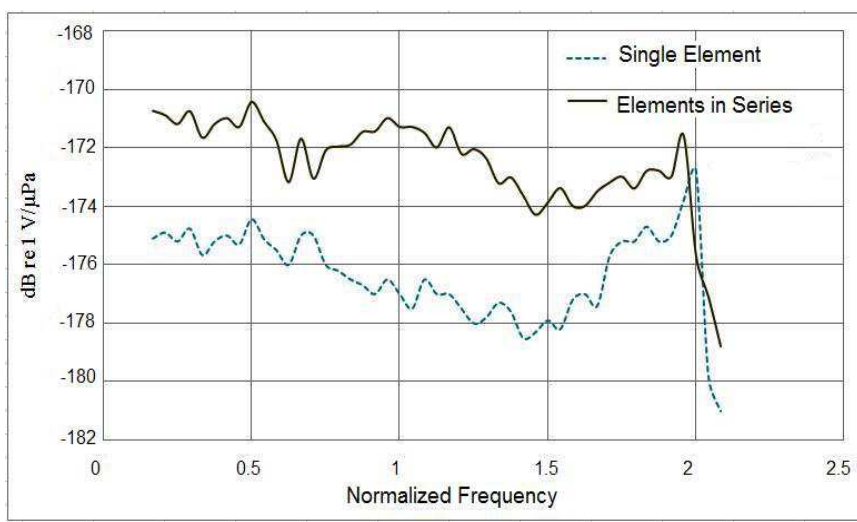

Figure 8. Measured sensitivity of $13 \mathrm{~mm}$ cymbal hydrophone array

The individual performance of a single element is also shown in the figure.8. A buffer is used between hydrophone and preamplifier to obtain impedance matching. A block diagram showing the arrangement of different components is given in Fig.9.

Hydrophone $\rightarrow$ Buffer $\rightarrow$ Amplifier (20 dB) $\rightarrow$ Filter

Figure 9. Block diagram showing the arrangement of different components

Earlier the author reported an RS of $\sim-205 \mathrm{~dB}$ re $1 \mathrm{~V} / \mu \mathrm{Pa} @ 1 \mathrm{~m}$ for a $3 \times 3$ array with standard cymbal dimensions of $12.7 \mathrm{~mm}$ and a cavity depth of $320 \mu \mathrm{m}$ [8]. Our group has also reported the performance of $5 \times 10$ rectangular array in series and parallel combinations for $19 \mathrm{~mm}$ cymbal transducers [9]. An improved performance with a single element having a cavity depth of $600 \mu \mathrm{m}$ and $\Phi$ $13 \mathrm{~mm}$ is very promising for future development of light weight thin hydrophone arrays for shallow water oceanographic applications. From the experimental result obtained by connecting the elements in series and the gain in $\mathrm{RS}$, it is well understood that identical element fabrication and procedure establishment is a critical factor in obtaining an excellent array performance as reported in this paper.

\section{Conclusions}

The in-air and in-water analysis have been carried out using finite element analysis software ATILA. Results of computation by finite element modeling were used to choose the dimensions of cymbal transducer. For achieving improved sensitivity, there should be minimum metal cap thickness combined with an optimum cavity depth. Emphasis is placed on the performance of the cymbal transducers with different cavity depth and performance difference of cymbals before and after encapsulation. Modeling results of the cymbal transducer with and without encapsulation show the effect of encapsulation on the performance of cymbal transducers. The in-air first resonance frequency shifted from $45 \mathrm{kHz}$ to $38 \mathrm{kHz}$ after the encapsulation and it has a pronounced effect on the frequency band of operation as a hydrophone. This is in principle applicable to all Cymbal type hydrophones/projector designs. Both $8 \mathrm{~mm}$ and $13 \mathrm{~mm}$ diameter cymbal transducer with different cavity depths has been modeled using ATILA and a $13 \mathrm{~mm}$ diameter cymbal transducer with 600 micron cavity depth is manufactured and the performances are studied. Since the receiving sensitivity is directly proportional to the volume of ceramics, it is observed that $8 \mathrm{~mm}$ cymbals are giving low value of RS compared to $13 \mathrm{~mm}$ cymbal transducer, though the frequency range has enhanced for $8 \mathrm{~mm}$. For the precision manufacturing of metal caps, a combination die have been designed and fabricated. The RS of $13 \mathrm{~mm}$ cymbal experimentally showed a maximum value of $-178 \mathrm{~dB}$ re $1 \mathrm{~V} / \mu \mathrm{Pa} @ 1 \mathrm{~m}$ with a suitable pre amplifier having a gain of $20 \mathrm{~dB}$ for a single element and nearly $6 \mathrm{~dB}$ gain is obtained when connected all the three elements in series. The array gain can be again improved by making identical elements with similar performances. This work is under progress.

\section{Acknowledgements}

The authors are grateful to other members of Marine Sensor Systems group for their keen support. This work is funded completely by National Institute of Ocean Technology (NIOT), Ministry of Earth Sciences, Govt. of India.

\section{References}

[1] J.F. Fernfindez , A. Dogan, J.T. Fielding, K. Uchino, R.E. Newnham "Tailoring the performance of ceramic-metal Piezo composite actuators, 'cymbals"' Sensors and Actuators, A 65 (1998) pp 228-237.

[2] R.E. Newnham, A. Dogan, D.C. Markley, J.F. Tressler, J. Zhang, E. Uzgur, R.J. Meyer,Jr., A.C. Hladky-Hennion and W. J.Hughes "Size Effects in Capped Ceramic Underwater Sound Projectors, IEEE OCEAN'02 MTS 4, (2002) PP 2315-2321.

[3] Denghua Li, Min Wu, Peixi Oyang, Xiaofei Xu, "Cymbal piezoelectric composite underwater acoustic transducer" Ultrasonics 44(2006) pp 685-687.

[4] E. Reissner, "On axi-symmetrical vibrations of shallow spherical shells," Quart. Appl. Math, 13 279, 1950.

[5] R.S. Woollet, "Theory of the piezoelectric flexural disk transducer with applications to underwater sound," USL Research Report No. 490, S-FOOI 03 04.1, US. Navy Underwater Sound Laboratoly, Fort Tmmbull, New London, CT, 1960.

[6] Cheng-Liang Sun, S.S.Guo , W.P.Li, Z.B.Xing, G.C.Liu, X.-Z.Zhao, "Displacement amplification and resonance characteristic of the cymbal transducers", Sensors and Actuators A121 (2005) pp 213-220.

[7] P.Ochoa, J.L.Pons, M.Villegas, J.F.Fernandez, "Effect of bonding layer on the electromechanical response of the cymbal metal-ceramic Piezo composite" Journal of the European Ceramic Society 27 (2007) pp 1143-1149.

[8] Dhilsha Rajapan, P.M. Rajeshwari and M.Sankar, K Trinath and N.S.Prasad. "Miniaturized underwater sensors for the 
realization of Conformal arrays", OCEANS'06, IEEE/MTS, May 16-19, Asia Pacific, Singapore (2006).

[9] R.Dhilsha, P. M. Rajeshwari, M Sankar "Underwater performance of a $5 \times 10$ cymbal array for oceanographic applications", Proceedings of International Conference in Ocean Engineering,(ICOE'09),Feb 1-5, IIT Madras, Chennai, India(2009).
[10] C. Kannan, PM Rajeshwari, Shibu Jacob et al., 'Effect of manufacturing procedure on the miniaturized Flextensional Transducers (Cymbals) and Hydrophone array performance', IEEE/MTS,OCEANS'11, June 6-9, Santander, Spain (2011) 